\title{
Copy-move forgery detection using combined features and transitive matching
}

\author{
Cong Lin · Wei Lu • Xinchao Huang · Ke \\ Liu · Wei Sun · Hanhui Lin · Zhiyuan Tan
}

Received: date / Accepted: date

\begin{abstract}
Recently, the research of Internet of Things (IoT) and Multimedia Big Data (MBD) has been growing tremendously. Both IoT and MBD have a lot of multimedia data, which can be tampered easily. Therefore, the research of multimedia forensics is necessary. Copy-move is an important branch of multimedia forensics. In this paper, a novel copy-move forgery detection scheme using combined features and transitive matching is proposed. First, SIFT and LIOP are extracted as combined features from the input image. Second, transitive matching is used to improve the matching relationship. Third, a filtering approach using image segmentation is proposed to filter out false matches. Fourth, affine transformations are estimated between these image patches. Finally, duplicated regions are located based on those affine transformations. The experimental results demonstrate that the proposed scheme can achieve much better detection results on the public database under various attacks.
\end{abstract}

Keywords Multimedia Big Data - Internet of Things · Multimedia forensics - Region duplication detection $\cdot$ Copy-move forgery $\cdot$ Image segmentation $\cdot$ LIOP

Cong Lin and Wei Lu and Xinchao Huang

School of Data and Computer Science, Guangdong Key Laboratory of Information Security Technology, Sun Yat-sen University, Guangzhou 510006, China. E-mail: lincong0310@gmail.com E-mail: luwei3@mail.sysu.edu.cn

Wei Sun and Ke Liu

School of Electronics and Information Technology, Key Laboratory of Information Technology (Ministry of Education), Sun Yat-sen University, Guangzhou 510006, China.

Wei Lu

State Key Laboratory of Information Security, Institute of Information Engineering, Chinese Academy of Sciences, Beijing 100093, China.

Cong Lin and Hanhui Lin

Center for Faculty Development and Educational Technology, Guangdong University of Finance and Economics, Guangzhou 510320, China.

Zhiyuan Tan

School of Computing, Edinburgh Napier University, Edinburgh, EH10 5DT, UK. E-mail: z.tan@napier.ac.uk 


\section{Introduction}

In recent years, Internet of Things (IoT) [7] and Multimedia Big Data (MBD) $[32,84]$ represent two appealing fields for many researchers [48, 50, 76, 82].Internet of Things (IoT) impart networked connectivity to everyday objects in the physical world [7]. Various electronic devices in IoT have generated huge multimedia data. Multimedia has become the "biggest big data", which is MBD. There are many information security problems of IoT and MBD, i.e., the multimedia of IoT or MBD is tampered. The related research is multimedia forensics, which is a science of acquiring, analyzing, extracting, interpreting and producing an evidence from a multimedia source in civil, criminal or corporate cases of administrative nature [51].

Multimedia forensics $[38,61,78,80,81]$ is an important domain of information security $[9,10,12,13,19-24,39]$. Both IoT and MBD $[17,18,28,30,42,46,47,58,62,65-71,75$, $77,79,83,85]$ have a lot of multimedia data. Therefore, the research of the multimedia forensics is very meaningful to IoT and MBD. The multimedia forensics can be divided into many branches, i.e., copy-move and splicing.

In a copy-move attack, one or more parts of an image are copied and pasted into another part of the same image [27]. The object of study of copy-move is multimedia data, many multimedia data make up MBD. Therefore, copy-move is an analysis and treatment of MBD. Many image Copy-Move Forgery Detection (CMFD) schemes [4, $5,16,25,27,29,33,34,44,49,64,72]$ have been proposed in recent years. According to Christlein et al. [15], commonly known copy-move detection schemes can be divided into two branches. The first one is the block-based schemes, an image is divided into fixedsize overlapping blocks, the each block is represented by a block descriptor, then those descriptors are sorted and matched. The main difference of the block-based schemes is their block features. Fridrich et al. [27] use the Discrete Cosine Transform (DCT) as block features. Popescu and Farid [53] use the Principal Component Analysis (PCA) as block features. Bashar et al. [5] propose a CMFD method using the Discrete Wavelet Transform (DWT) or the Kernel Principal Component Analysis (KPCA). An improved DCT-based method is proposed by Huang et al. [34]. Bravo-Solorio and Nandi [8] propose a CMFD scheme based on the Fourier Transform. Li et al. [41] use the Polar Cosine Transform (PCT) as block features. Ryu et al. $[55,56]$ propose a CMFD scheme using Zernike moment, and Locality Sensitive Hashing (LSH) matching is adopted in [55]. A histogram of orientated gradients is applied to each block in [36]. A fast Walsh-Hadamard Transform (FWHT) is adopted in [73].

The block-based schemes are not robust to scale, rotation, JPEG compression and additive noise. So keyponint-based schemes are proposed. Feature exaction methods such as the Scale-Invariant Feature Transform (SIFT) [45] and the Speeded Up Robust Features (SURF) [6] are most widely used in keypoint-based schemes. Pan and Lyu [52] propose a framework of the keypoint-based schemes, and their feature was also SIFT. Amerini et al. [3] propose a method using SIFT feature, the g2NN matching and the Agglomerative Hierarchical Clustering (AHC). Shivakumar and Baboo [57] propose a scheme based on SURF and KD-Tree. Silva et al. [59] construct a multi-scale image representation and a voting process among all detection maps. A rotation invariance scheme is proposed by Christlein et al. [14]. The Harris corner points [31] in an image are detected in [11], and their description is based on step sector statistics. Li et al. [40] propose a scheme using the Maximally Stable Color Region (MSCR). Yang et al. [74] propose a scheme using KAZE [2] and SIFT [45]. The image segmentation is adopted by Li et al. [37] and Pun et al. [54]. The image is segmented by Simple Linear Iterative 
Clustering (SLIC) algorithm [1] before feature extraction. Lin et al. [43] propose a Keypoint Contexts (KC) scheme to deal with duplicated regions with few keypoints. Jin and Wang [35] use OpponentSIFT and optimized J-Linkage to detect duplicated regions.

The block-based scheme is not robust and the keypoint-based scheme cannot detect duplicated regions with few keypoints. To overcome this issue, in this paper, a novel copy-move forgery detection scheme using combined features and transitive matching is proposed.

The remainder of this paper is organized into three sections. Section 2 shows the framework of the proposed scheme and then explains each step in detail. To validate the effectiveness of the proposed scheme, the experimental results are given in Section 3. Finally, Section 4 draws conclusions.

\section{The proposed scheme}

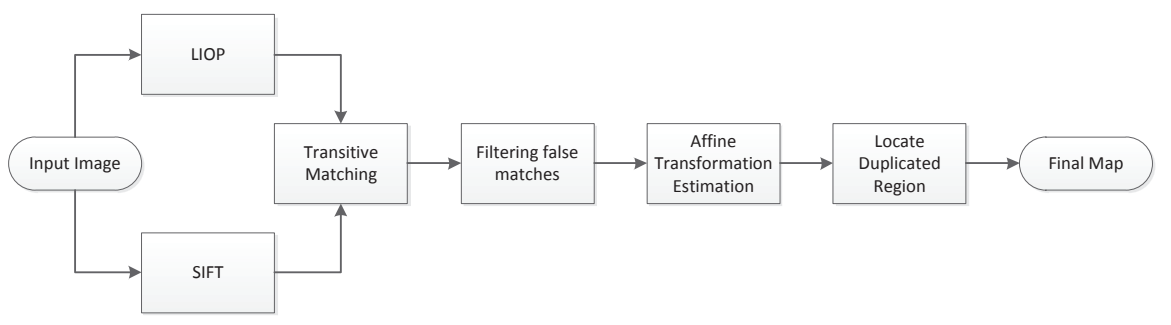

Fig. 1 The framework of the copy-move forgery detection scheme.
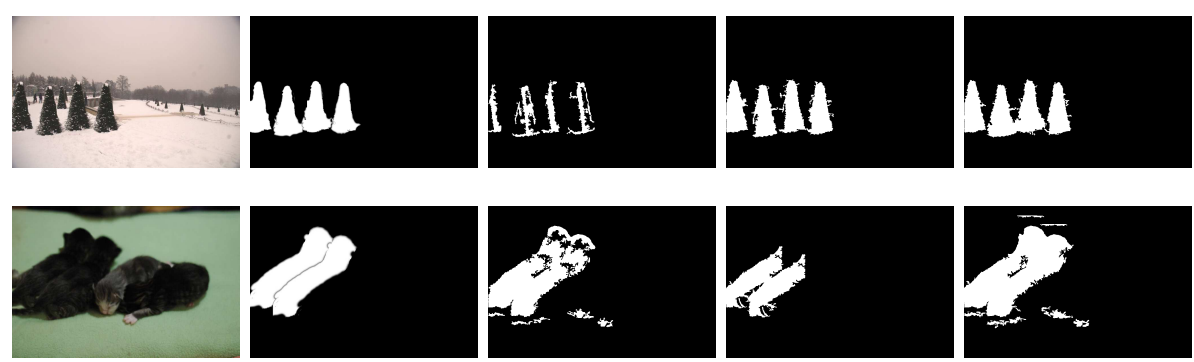

Fig. 2 Copy-move forgery detection results of the proposed scheme. Column 1: the forged images; column 2: the ground truth; column 3: the detection results only using SIFT; column 4: the detection results only using LIOP; column 5: the detection results using the proposed scheme(SIFT+LIOP). 
A block-based scheme is good at plain copy-move, but it cannot deal with significant geometrical transformations. A keypoint-based scheme is more robust than a blockbased scheme, but it cannot deal with duplicated regions with few keypoints. Therefore, a strategy of combined features is proposed by our scheme, where both the Local Intensity Order Pattern (LIOP) [63] and the Scale Invariant Feature Transform (SIFT) [45] are adopted as our combined features.

Now we describe the reason why we choose LIOP and SIFT as the combined features. First, SIFT is invariant to image scale, rotation, addition of noise, etc. Meanwhile, SIFT has been widely used in many CMFD schemes $[3,4,52]$ and obtained good results. Second, both local and overall intensity ordinal information of the local patch are captured by the LIOP descriptor [63]. Therefore, LIOP is invariant to image scale, rotation, viewpoint change, image blur and JEPG compression. We choose combined features to deal with duplicated regions with few keypoints.

We are familiar with SIFT. So let's introduce LIOP [63]. The main idea of LIOP is that when the intensity monotonous changes, the relative order of pixel intensities remains unchanged. The steps of LIOP are as follows. First, the local patch is divided into ordinal bins using the overall intensity order. Second, for a point $\mathrm{x}$, the LIOP of which is defined as follows [63]:

$$
\operatorname{LIOP}(x)=\Phi(\gamma(P(x)))
$$

where $P(x)=\left(I\left(x_{1}\right), I\left(x_{2}\right), \cdots, I\left(x_{N}\right)\right) \in P^{N}$ and $I\left(x_{i}\right)$ represent the intensity of the $i$-th neighboring sample point $x_{i}$. Third, for a local patch, to accumulate the LIOPs of points in each ordinal bin, we obtained the LIOP descriptor [63]:

$$
\begin{array}{r}
D_{L I O P}=\left(\operatorname{des}_{1}, \operatorname{des}_{2}, \cdots, \operatorname{des}_{B}\right) \\
\operatorname{des}_{i}=\Sigma_{x \in b i n_{i}} \omega(x) \operatorname{LIOP}(x)
\end{array}
$$

where $\omega(x)$ is a weighting function and B is the number of the ordinal bins.

In some cases, the results of LIOP are better than that of SIFT. But in other cases, the results of SIFT are better than that of LIOP. Therefore, both LIOP and SIFT are integrated as our combined features, and the results of combined features are better than that of LIOP or SIFT, as shown in Fig. 2.

\subsection{Transitive matching}

The detected keypoints are tentatively matched using their feature vectors. There are two common matching methods. The first one is the $2 \mathrm{NN}$ matching proposed by Pan and Lyu [52]. Given a keypoint, its distance $d_{1}$ to the nearest neighbor and the distance $d_{2}$ to the next-nearest-neighbour are compared, if $d_{1} / d_{2}$ is less than a threshold (often fixed to 0.5 or 0.6 ), a pair of keypoints is obtained. To deal with multiple keypoint matching, Amerini et al. [3] proposed the generalized 2NN (g2NN) matching.

Some duplicated regions which are copied and pasted more than once still cannot be detected by the g2NN matching, because some matched keypoints cannot be detected. Therefore, the transitive matching is proposed to improve the matching relationship. We obtain a list of matched keypoints after the g2NN matching, as shown in Fig. 3, there are three duplicated regions, which are labeled as $\Omega_{1}, \Omega_{2}$ and $\Omega_{3}$. The duplicated regions $\Omega_{1}$ and $\Omega_{3}$ are easy to be detected for there are enough matched keypoints between them. Neither the matched keypoints between $\Omega_{1}$ and $\Omega_{2}$, nor the matched 


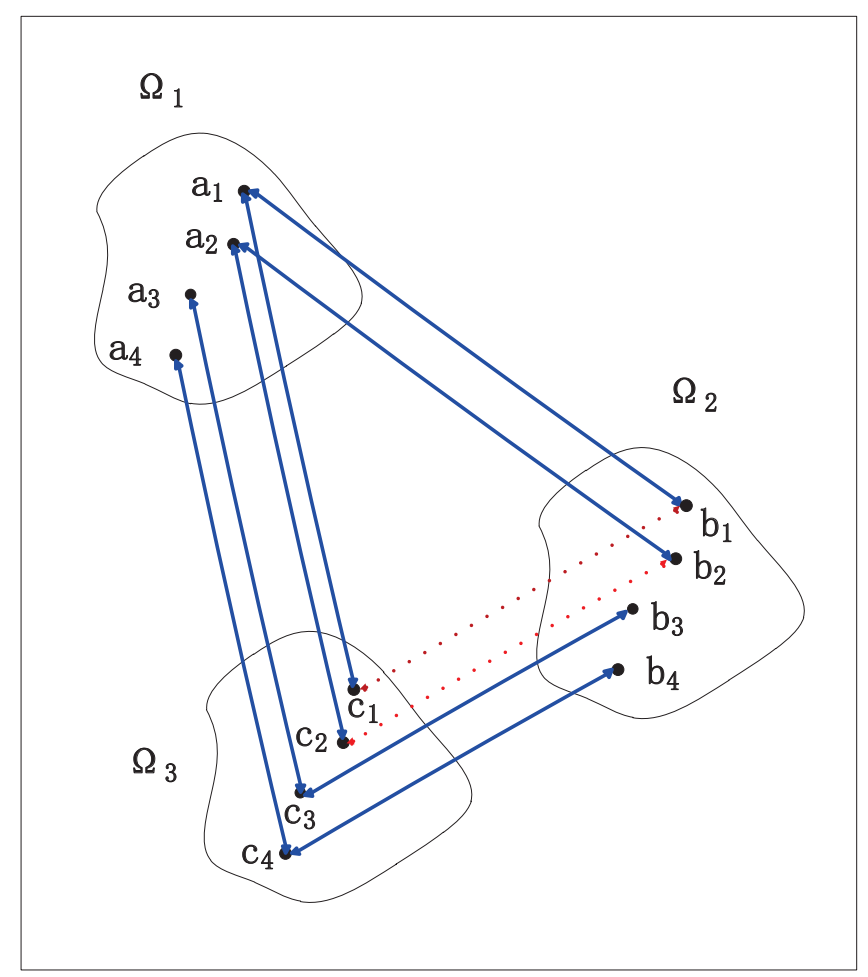

Fig. 3 The transitive matching. There are three duplicated regions, such as $\Omega_{1}, \Omega_{2}$ and $\Omega_{3}$. The initial matching are connected by a solid line, for instance, $\left(a_{1}, b_{1}\right)$ and $\left(a_{1}, c_{1}\right)$. The transitive matching are connected by a dotted line, for instance, $\left(b_{1}, c_{1}\right)$.

keypoints between $\Omega_{2}$ and $\Omega_{3}$ are sufficient. So the duplicated region $\Omega_{2}$ cannot be detected.

In fact, keypoints are sufficient, only their matching relationship is not detected. Now the transitive matching is used to obtain the new matching relationship. We obtain the matched keypoints $\left(a_{1}, c_{1}\right)$ between $\Omega_{1}$ and $\Omega_{3}$, the matched keypoints $\left(a_{1}, b_{1}\right)$ between $\Omega_{1}$ and $\Omega_{2}$, which are connected by a solid line in Fig. 3. Keypoints $a_{1}$ is matched with $c_{1}$, and the same keypoints is matched with $b_{1}$, then we draw a conclusion that keypoints $b_{1}$ is matched with $c_{1}$, which is the transitive matching. Therefore, the transitive matching can be described as follows:

$$
\left(K_{1}, K_{2}\right),\left(K_{1}, K_{3}\right) \Rightarrow\left(K_{2}, K_{3}\right)
$$

where $\left(K_{1}, K_{2}\right)$ indicates the matched keypoints $K_{1}$ and $K_{2}$. Then the new matched keypoints such as $\left(b_{1}, c_{1}\right)$ and $\left(b_{2}, c_{2}\right)$ is obtained, which are connected by a dotted line in Fig. 3. Thus, we can estimate the affine transformation between $\Omega_{2}$ and $\Omega_{3}$ after the transitive matching. The transitive matching try to detect a region which is copied and pasted more than once. The matching relation is improved by the transitive matching. To decrease mismatches, the transitive matching is limited to some regions which have matching relation. As shown in Fig. 3, there are matched keypoints between the three regions, which are connected by a solid line, then the transitive matching is carried out in the three regions. 


\subsection{Filtering false matches}

In the section, the filtering algorithm to discard false matches is described. To improve the accuracy of affine transformations, those mismatched keypoints should be discarded after the transitive matching. Therefore, the Random Sample Consensus (RANSAC) algorithm [26] is adopted by Pan and Lyu [52]. The RANSAC algorithm returns with the affine transformations that lead to the largest number of matched keypoints and the smallest error. Some mismatched keypoints can be discarded by RANSAC. But when there are lots of mismatched keypoints, the inaccurate affine transformation will be obtained by RANSAC. To overcome this issue, some false matches should be filtered, and the corresponding affine transformation will not be estimated. Considering the duplicated regions are usually meaningful regions, the input image is divided into non-overlapping image patches. It should be noted that the images are segmented by the Simple Linear Iterative Clustering (SLIC) algorithm [1]. Then $N_{m}$ is adopted to represent the number of matched keypoints between the two image patches. If $N_{m}$ is larger than a threshold, an affine transformation between the two image patches is estimated. Otherwise, those mismatched keypoints will be discarded. Thus some false matches can be discarded by our filtering algorithm.

\subsection{Estimation of affine transformation}

After the matched keypoints and the image patches are obtained, an affine transformation is estimated between the two image patches, one denotes as the source region and the other denotes as the forged region, if there are more than three matched keypoint between the two image patches. Two matched keypoints $\hat{x}_{i}=\left(x_{i}, y_{i}, 1\right)^{T}$ and $\hat{x}_{i}^{\prime}=\left(x_{i}^{\prime}, y_{i}^{\prime}, 1\right)^{T}$ are from the source region and the forged region, respectively. Formally, their transformation can be expressed in matrix form as:

$$
\hat{x}_{i}^{\prime}=H \hat{x}_{i}=\left(\begin{array}{ccc}
h_{11} & h_{12} & t_{x} \\
h_{21} & h_{22} & t_{y} \\
0 & 0 & 1
\end{array}\right) \hat{x}_{i}
$$

where $t_{x}$ and $t_{y}$ are denoted as the translation factors, while $h_{11}, h_{12}, h_{21}$ and $h_{22}$ are denoted as rotation and scaling directions deformation. An affine transformation has six degrees of freedom, corresponding to the six matrix elements, then the transformation can be computed from three pairs of matched keypoints that are not collinear. Using RANSAC, the transformation matrix which returns the the largest number matched keypoints is obtained. Meanwhile, their total error of the affine transformation is minimized. Thus, an affine transformation between the two image patches is estimated. Then the duplicated regions are located according to the affine transformation [52].

\section{Experiments and discussions}

\subsection{Dataset and error measures}

To evaluate the efficiency of the proposed scheme, the Image Manipulation Dataset (IMD) [15] is adopted as the image dataset. The average size of an image is about 
Table 1 Setting of the attacks on IMD

\begin{tabular}{lcc}
\hline Attacks & Criteria & Parameters \\
\hline Scaling & Ratio & $0.91: 0.02: 1.09$ \\
Rotation & Angle & $2^{\circ}: 2^{\circ}: 10^{\circ}$ \\
AWGN & Stand Deviation & $0.02: 0.02: 0.1$ \\
JPEG & Quality Factor & $20: 10: 100$ \\
\hline
\end{tabular}

$3000 \times 2300$ pixels. There are 1488 images on IMD. The details of the utilized image dataset are shown in Table 1.

In fact, the forgery is more difficult to be detected when the duplicated regions are small. Many images on the Internet are usually small, they are not as big as the images on IMD. Therefore, all the images on IMD are resized, just as Li et al. [37] did. The maximum of the width and the height of the images are set to 800 pixels. The proposed scheme is rather challenging for the duplicated regions are difficult to be detected after the images are resized.

It should be noted that the images on IMD are segmented by the SLIC algorithm [1], which is implemented by vlFeat library [60], where all the images on IMD are empirically divided into 100 image patches.

To assess the proposed scheme, we should test the detection error at two different levels, namely the image level and the pixel level. The detection error are measured by the recall, the precision, and the $F_{1}$ score [15], which are calculated as follows:

$$
\begin{gathered}
\text { precision }=\frac{\mid\{\text { Forged pixels }\} \cap\{\text { Detected pixels }\} \mid}{\mid\{\text { Detected pixels }\} \mid} \\
\text { recall }=\frac{\mid\{\text { Forged pixels }\} \bigcap\{\text { Detected pixels }\} \mid}{\mid\{\text { Forged pixels }\} \mid} \\
F_{1}=\frac{2 * \text { precision } * \text { recall }}{\text { precision }+ \text { recall }}
\end{gathered}
$$

\subsection{Comparisons with other relevant methods}

In the section, the proposed scheme is compared with several state-of-the-art existing schemes, for instance, SIFT [3,52], SURF [57], JLinkage [4] and Zernike [56]. The results of SIFT, SURF and Zernike are different with Christlein et al. [15] because of the image resizing. The process of resizing will make the duplicated regions smaller than before. Therefore, it will difficult to be detected for all the CMFD schemes. The proposed scheme combines both LIOP and SIFT. Some detection results of the proposed scheme in comparison with only SIFT or LIOP are shown in Fig. 2. Obviously, the most duplicated regions can be detected by the the proposed scheme.

\subsubsection{Detection results under plain copy-move}

In this section, we evaluate the proposed scheme under ideal conditions. There are 48 original images and 48 forgery images, in which a one-to-one copy-move is implemented. The experimental results under plain copy-move at the image level and the pixel level are shown in Table 2 and Table 3, respectively. It should be noted that all the images on IMD are resized and the experimental results are different with Christlein et al. [15]. From Table 2 and Table 3, it can be observed easily that the recall of the proposed 
scheme is the best among all the test schemes. The precision of the proposed scheme is better than that of SIFT, SURF and JLinkage, all of which are keypoint-based schemes. Meanwhile, the $F_{1}$ score of the proposed scheme is much better than that of the existing state-of-the-art schemes. As a comprehensive evaluation, the $F_{1}$ score combines both the recall and the precision into a single value. Therefore, the proposed scheme is the best among the existing state-of-the-art schemes.

Table 2 Detection results for plain copy-move at the image level

\begin{tabular}{l|ccc}
\hline Methods & recall $(\%)$ & precision $(\%)$ & $F_{1}(\%)$ \\
\hline SIFT [3,52] & 47.92 & 74.19 & 58.23 \\
SURF [57] & 43.75 & 72.41 & 54.55 \\
JLinkage [4] & 62.50 & 78.95 & 69.77 \\
Zernike [56] & 79.17 & 88.37 & 83.52 \\
Proposed & 93.75 & 81.82 & 87.38 \\
\hline
\end{tabular}

Table 3 Detection results for plain copy-move at the pixel level

\begin{tabular}{l|ccc}
\hline Methods & recall $(\%)$ & precision $(\%)$ & $F_{1}(\%)$ \\
\hline SIFT [3,52] & 37.93 & 36.79 & 37.35 \\
SURF [57] & 25.81 & 31.44 & 28.35 \\
JLinkage [4] & 47.47 & 48.12 & 47.79 \\
Zernike [56] & 53.92 & 87.37 & 66.68 \\
Proposed & 75.41 & 73.44 & 74.42 \\
\hline
\end{tabular}

\subsubsection{Detection results under other attackers}

This section presents the comparison of the proposed method with other schemes under various attacks. The proposed scheme is evaluated by the recall, the precision and the $F_{1}$ at the pixel level. It should be noted that the results of SIFT, SURF and Zernike are different with Christlein et al. [15] because of the image resizing. In the experiments, all the images are resized to no more than 800 pixels, just as Li et al. [37] did.

Figure 4 shows the recall results of the proposed scheme compared with the test schemes. It can be observed easily that the recall of the proposed scheme is the best among all the test schemes, which means that more number of duplicated regions can be obtained by the proposed scheme.

Figure 5 shows the precision results of the proposed scheme compared with the test schemes. The precision results of the proposed scheme is better than that of SIFT, SURF and JLinkage, all of which are keypoint-based schemes. As a block-based scheme, the precision results of Zernike is the best among all the test schemes. Therefore, the precision results of the proposed scheme is the best among all the keypoint-based schemes.

Figure 6 shows the $F_{1}$ results of the proposed scheme compared with the test schemes. Obviously, the proposed scheme outperforms the prior arts in terms of $F_{1}$ criterion. The $F_{1}$ score combines both the precision and the recall into a single value, it is a comprehensive evaluation. Therefore, the proposed scheme is better than the existing state-of-the-art schemes under various attacks. 


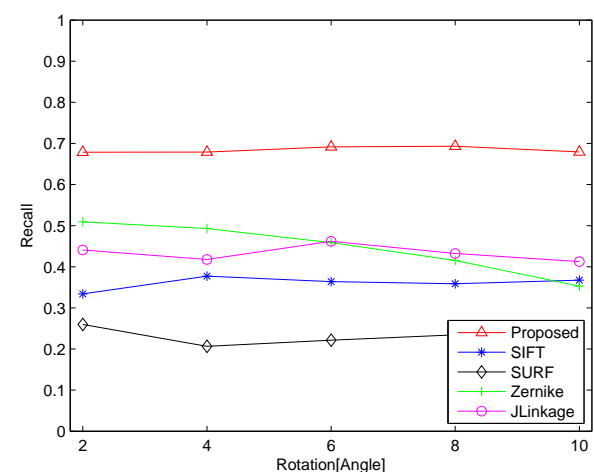

(a)

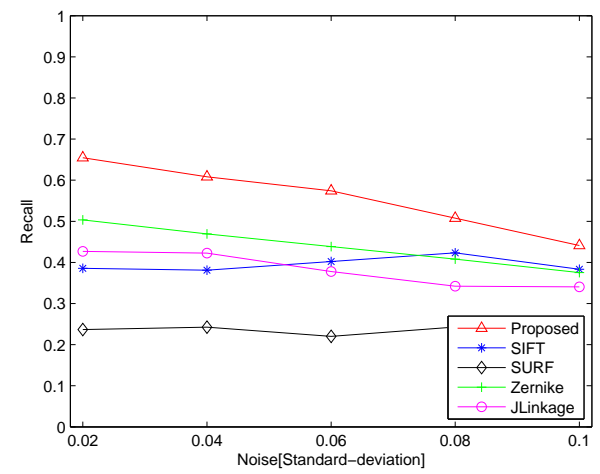

(c)

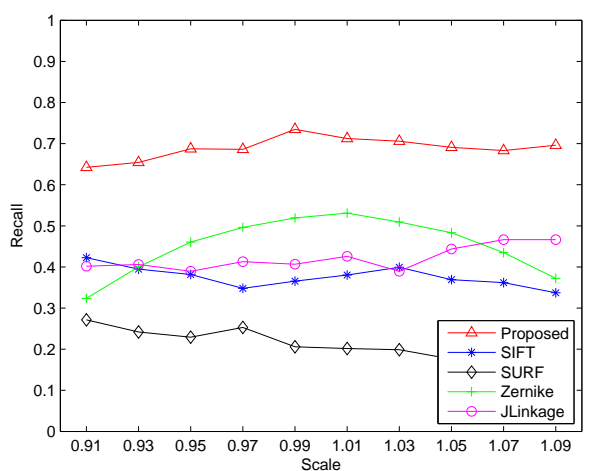

(b)

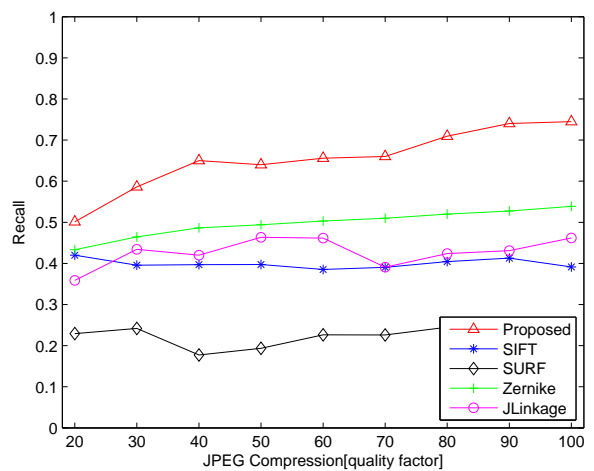

(d)

Fig. 4 Recall results at the pixel level. (a) Rotation, (b) Scale, (c) Adding noise, (d) JPEG compression.

\section{Conclusions}

In this paper, a novel copy-move forgery detection scheme using combined features and transitive matching is proposed. The specific contributions are summarized as follows. First, combined features which are composed of LIOP and SIFT are proposed. Thus, some duplicated regions with few keypoints can be detected. Second, transitive matching is used after the g2NN matching, then the matching relationship is improved. Third, to discard the false matches, a new filtering approach based on image segmentation is proposed. Experimental results show that the proposed scheme can achieve the best recall and the best $F_{1}$ score under challenging conditions.

\section{Acknowledgements}

This work is supported by the National Natural Science Foundation of China (No. U1736118), the Natural Science Foundation of Guangdong (No. 2016A030313350), the Special Funds for Science and Technology Development of Guangdong (No. 2016KZ010103), the Key Project of Scientific Research Plan of Guangzhou (No. 201804020068), the 


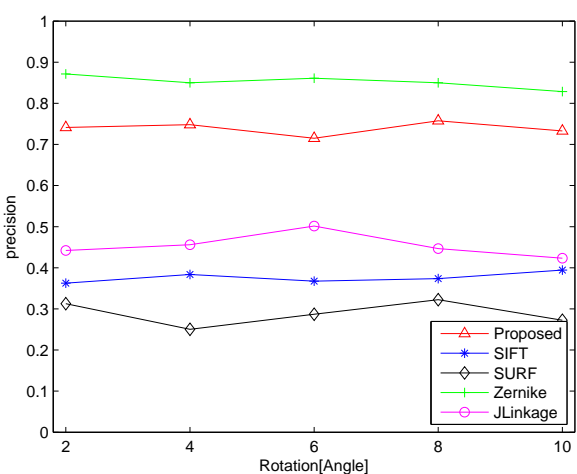

(a)

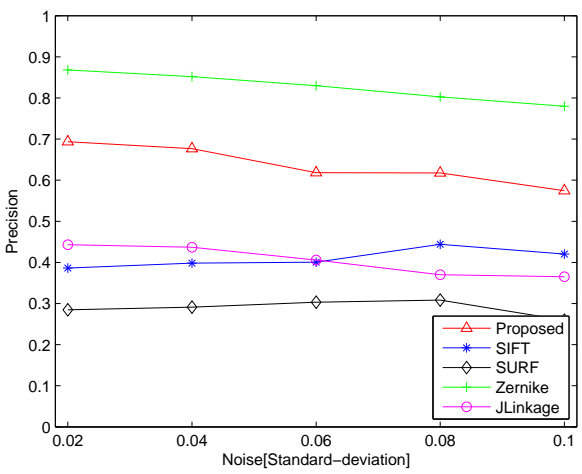

(c)

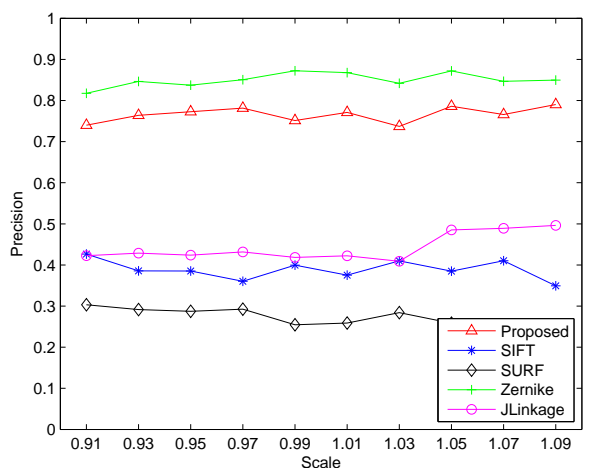

(b)

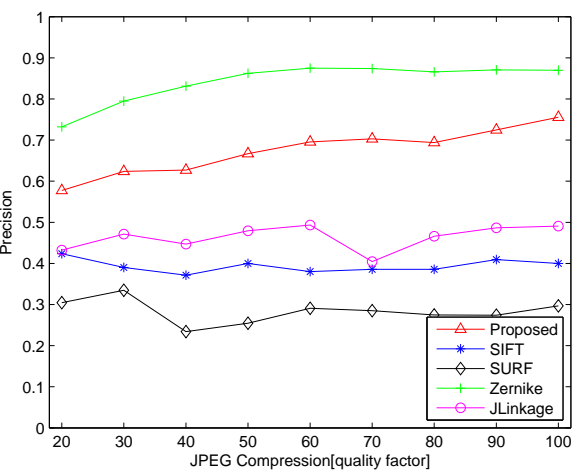

(d)

Fig. 5 Precision results at the pixel level. (a) Rotation, (b) Scale, (c) Adding noise, (d) JPEG compression.

Fundamental Research Funds for the Central Universities (No. 16lgjc83 and No. 17lgjc45), the Science and Technology Planning Project of Guangdong Province (Grant No.2017A040405051).

\section{References}

1. Achanta, R., Shaji, A., Smith, K., Lucchi, A., Fua, P., Süsstrunk, S.: Slic superpixels compared to state-of-the-art superpixel methods. IEEE Transactions on Pattern Analysis and Machine Intelligence 34(11), 2274-2282 (2012)

2. Alcantarilla, P.F., Bartoli, A., Davison, A.J.: Kaze features. In: European Conference on Computer Vision(ECCV), pp. 214-227. Florence, Italy (2012)

3. Amerini, I., Ballan, L., Caldelli, R., Bimbo, A.D., Serra, G.: A SIFT-based forensic method for copy-move attack detection and transformation recovery. IEEE Transactions on Information Forensics and Security 6(3), 1099-1110 (2011)

4. Amerini, I., Ballan, L., Caldelli, R., Bimbo, A.D., Tongo, L.D., Serra, G.: Copy-move forgery detection and localization by means of robust clustering with J-Linkage. Signal Processing: Image Communication 28(6), 659-669 (2013)

5. Bashar, M., Noda, K., Ohnishi, N., Mori, K.: Exploring duplicated regions in natural images. IEEE Transactions on Image Processing PP(99), 1-1 (2010)

6. Bay, H., Ess, A., Tuytelaars, T., Gool, L.V.: SURF: Speeded up robust features. Computer Vision and Image Understanding 110(3), 346-359 (2008) 


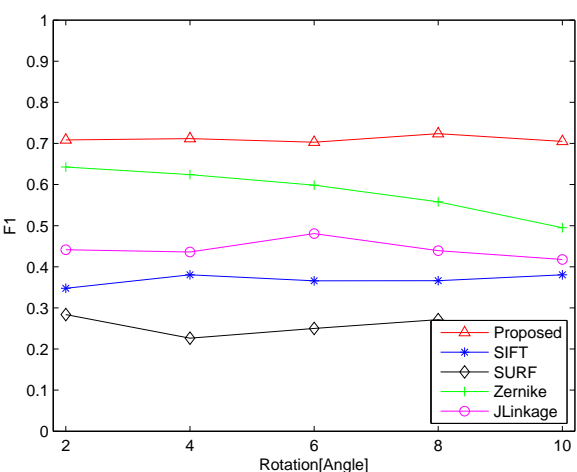

(a)

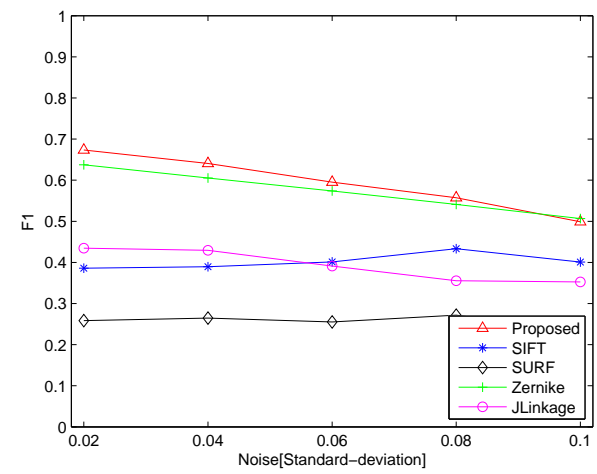

(c)

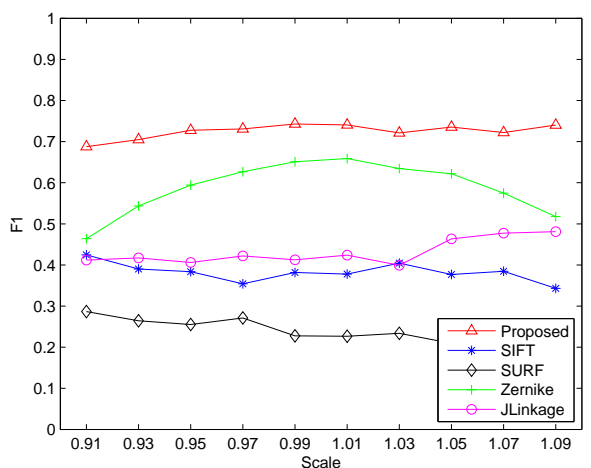

(b)

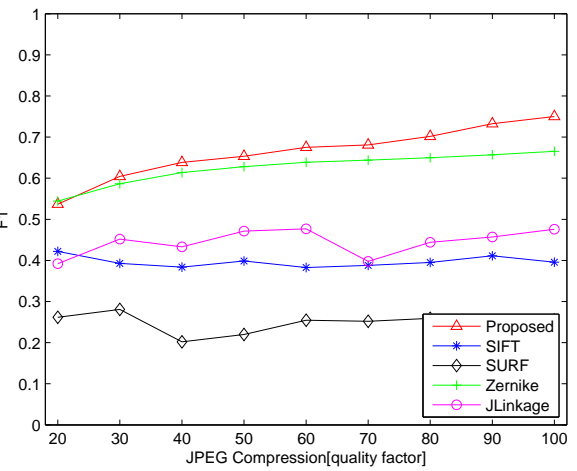

(d)

Fig. $6 F_{1}$ results at the pixel level. (a) Rotation, (b) Scale, (c) Adding noise, (d) JPEG compression.

7. Bedi, G., Venayagamoorthy, G.K., Singh, R., Brooks, R., Wang, K.C.: Review of internet of things (iot) in electric power and energy systems. IEEE Internet of Things Journal PP(99), 1-1 (2018)

8. Bravo-Solorio, S., Nandi, A.K.: Exposing duplicated regions affected by reflection, rotation and scaling. In: IEEE International Conference on Acoustics, Speech and Signal Processing(ICASSP), pp. 1880-1883. Prague, Czech Republic (2011)

9. Chen, J., Lu, W., Fang, Y., Liu, X., Yeung, Y., Yingjie, X.: Binary image steganalysis based on local texture pattern. Journal of Visual Communication and Image Representation 55, $149-156(2018)$

10. Chen, J., Lu, W., Yeung, Y., Xue, Y., Liu, X., Lin, C., Zhang, Y.: Binary image steganalysis based on distortion level co-occurrence matrix. Computers, Materials and Continua 55(2), 201-211 (2018)

11. Chen, L., Lu, W., Ni, J., Sun, W., Huang, J.: Region duplication detection based on harris corner points and step sector statistics. Journal of Visual Communication and Image Representation 24(3), 244-254 (2013)

12. Chen, X., Weng, J., Lu, W., Xu, J.: Multi-gait recognition based on attribute discovery. IEEE Transactions on Pattern Analysis and Machine Intelligence PP(99), 1-1 (2018)

13. Chen, X., Weng, J., Lu, W., Xu, J., Weng, J.: Deep manifold learning combined with convolutional neural networks for action recognition. IEEE Transactions on Neural Networks and Learning Systems PP(99), 1-15 (2017)

14. Christlein, V., Riess, C., Angelopoulou, E.: On rotation invariance in copy-move forgery detection. In: IEEE International Workshop on Information Forensics and Security (WIFS), pp. 1-6. Seattle, WA, USA (2010) 
15. Christlein, V., Riess, C., Jordan, J., Riess, C., , Angelopoulou, E.: An evaluation of popular copy-move forgery detection approaches. IEEE Transactions on Information Forensics and Security $\mathbf{7}(6), 1841-1854$ (2012)

16. Cozzolino, D., Poggi, G., Verdoliva, L.: Efficient dense-field copy-move forgery detection. IEEE Transactions on Information Forensics and Security 10(11), 2284-2297 (2015)

17. Fang, W., Li, Y., Zhang, H., Xiong, N., Lai, J., Vasilakos, A.V.: On the throughput-energy tradeoff for data transmission between cloud and mobile devices. Information Sciences 283(283), 79-93 (2014)

18. Fang, Y., Fang, Z., Yuan, F., Yang, Y., Yang, S., Xiong, N.N.: Optimized multioperator image retargeting based on perceptual similarity measure. IEEE Transactions on Systems Man and Cybernetics Systems 47(11), 2956-2966 (2017)

19. Feng, B., Lu, W., Sun, W.: Secure binary image steganography based on minimizing the distortion on the texture. IEEE Transactions on Information Forensics and Security 10(2), 243-255 (2014)

20. Feng, B., Lu, W., Sun, W.: Binary image steganalysis based on pixel mesh markov transition matrix. Journal of Visual Communication and Image Representation 26, 284-295 (2015)

21. Feng, B., Lu, W., Sun, W.: Novel steganographic method based on generalized k-distance n-dimensional pixel matching. Multimedia Tools and Applications 74(21), 9623-9646 (2015)

22. Feng, B., Lu, W., Sun, W., Huang, J., Shi, Y.Q.: Robust image watermarking based on tucker decomposition and adaptive-lattice quantization index modulation. Signal Processing: Image Communication 41(C), 1-14 (2016)

23. Feng, B., Weng, J., Lu, W., Pei, B.: Steganalysis of content-adaptive binary image data hiding. Journal of Visual Communication and Image Representation 46, 119-127

24. Feng, B., Weng, J., Lu, W., Pei, B.: Multiple watermarking using multilevel quantization index modulation. In: International Workshop on Digital Watermarking, pp. 312-326. Beijing, China (2017)

25. Ferreira, A., Felipussi, S.C., Alfaro, C., Fonseca, P., Vargasmunoz, J.E., Dos Santos, J.A., Rocha, A.: Behavior knowledge space-based fusion for copy-move forgery detection. IEEE Transactions on Image Processing 25(10), 4729-4742 (2016)

26. Fischler, M.A., Bolles, R.C.: Random sample consensus: A paradigm for model fitting with applications to image analysis and automated cartography. Communications of the ACM 24(6), 381-395 (1981)

27. Fridrich, J., Soukal, D., Lukáš, J.: Detection of copy-move forgery in digital images. In: Proceeding of Digital Forensic Research Workshop, pp. 19-23. Cleveland, OH, USA (2003)

28. Gao, L., Yu, F., Chen, Q., Xiong, N.: Consistency maintenance of do and undo/redo operations in real-time collaborative bitmap editing systems. Cluster Computing 19(1), 255-267 (2016)

29. Ghorbani, M., Firouzmand, M., Faraahi, A.: DWT-DCT (QCD) based copy-move image forgery detection. In: International Conference on Systems, Signals and Image Processing, pp. 1-4. Sarajevo (2011)

30. Gui, J., Hui, L., Xiong, N.: A game-based localized multi-objective topology control scheme in heterogeneous wireless networks. IEEE Access 5(99), 2396-2416 (2017)

31. Harris, C.G., Stephens, M.J.: A combined corner and edge detector. In: Alvey Vision Conference, pp. 147-151 (1988)

32. Hu, C., Xu, Z., Liu, Y., Mei, L., Chen, L., Luo, X.: Semantic link network-based model for organizing multimedia big data. IEEE Transactions on Emerging Topics in Computing 2(3), 376-387 (2014)

33. Huang, H., Guo, W., Zhang, Y.: Detection of copy-move forgery in digital images using SIFT algorithm. In: IEEE Pacific-Asia Workshop on Computational Intelligence and Industrial Application, pp. 272-276 (2008)

34. Huang, Y., Lu, W., Sun, W., Long, D.: Improved DCT-based detection of copy-move forgery in images. Forensic Science International 206(1-3), 178-184 (2011)

35. Jin, G., Wan, X.: An improved method for SIFT-based copy-move forgery detection using non-maximum value suppression and optimized J-Linkage. Signal Processing: Image Communication 57, 113-125 (2017)

36. Lee, J.C., Chang, C.P., Chen, W.K.: Detection of copy-move image forgery using histogram of orientated gradients. Information Sciences 321(C), 250-262 (2015)

37. Li, J., Li, X., Yang, B., Sun, X.: Segmentation-based image copy-move forgery detection scheme. IEEE Transactions on Information Forensics and Security 10(3), 507-518 (2015) 
38. Li, J., Lu, W.: Blind image motion deblurring with L0-regularized priors. Journal of Visual Communication and Image Representation 40, 14-23 (2016)

39. Li, J., Lu, W., Weng, J., Mao, Y., Li, G.: Double jpeg compression detection based on block statistics. Multimedia Tools and Applications pp. 1-16 (2018)

40. Li, J., Yang, F., Lu, W., Sun, W.: Keypoint-based copy-move detection scheme by adopting mscrs and improved feature matching. Multimedia Tools and Applications 76(20), 1-15 (2016)

41. Li, Y.: Image copy-move forgery detection based on polar cosine transform and approximate nearest neighbor searching. Forensic Science International 224(1-3), 59 (2013)

42. Lin, B., Guo, W., Xiong, N., Chen, G., Vasilakos, A.V., Zhang, H.: A pretreatment workflow scheduling approach for big data applications in multicloud environments. IEEE Transactions on Network and Service Management 13(3), 581-594 (2016)

43. Lin, C., Lu, W., Sun, W., Zeng, J., Xu, T., Lai, J.H.: Region duplication detection based on image segmentation and keypoint contexts. Multimedia Tools and Applications (11), $1-18$ (2017)

44. Liu, G., Wang, J., Lian, S., Wang, Z.: A passive image authentication scheme for detecting region-duplication forgery with rotation. Journal of Network and Computer Applications 34(5), 1557-1565 (2011)

45. Lowe, D.G.: Distinctive image features from scale-invariant keypoints. International Journal of Computer Vision 60(2), 91-110 (2004)

46. Lu, X., Tu, L., Zhou, X., Xiong, N., Sun, L.: Vimedianet: an emulation system for interactive multimedia based telepresence services. Journal of Supercomputing 73(8), 3562-3578 (2017)

47. Lu, Z., Lin, Y.R., Huang, X., Xiong, N., Fang, Z.: Visual topic discovering, tracking and summarization from social media streams. Multimedia Tools and Applications 76(8), 1-25 (2017)

48. Ma, Y., Luo, X., Li, X., Bao, Z., Zhang, Y.: Selection of rich model steganalysis features based on decision rough set $\alpha$-positive region reduction. IEEE Transactions on Circuits and Systems for Video Technology PP(99), 1-1 (2018)

49. Mahdian, B., Saic, S.: Detection of copy-move forgery using a method based on blur moment invariants. Forensic Science International 171, 180-189 (2007)

50. Melro, L.S., Jensen, L.R.: Influence of functionalization on the structural and mechanical properties of graphene. Computers Materials and Continua 53(2), 111-131 (2017)

51. Nelson, B., Phillips, A., Steuart, C.: Guide to Computer Forensics and Investigations. Delmar Learning (2015)

52. Pan, X., Lyu, S.: Region duplication detection using image feature matching. IEEE Transactions on Information Forensics and Security 5(4), 857-867 (2010)

53. Popescu, A.C., Farid, H.: Exposing digital forgeries by detecting duplicated image regions. Tech. Rep. TR2004-515, Department of Computer Science, Dartmouth College (2004)

54. Pun, C.M., Yuan, X.C., Bi, X.L.: Image forgery detection using adaptive over-segmentation and feature points matching. IEEE Transactions on Information Forensics and Security 10(8), 1705-1716 (2015)

55. Ryu, S.J., Kirchner, M., Lee, M.J., Lee, H.K.: Rotation invariant localization of duplicated image regions based on Zernike moments. IEEE Transactions on Information Forensics and Security 8(8), 1355-1370 (2013)

56. Ryu, S.J., Lee, M.J., Lee, H.K.: Detection of copy-rotate-move forgery using Zernike moments. In: IEEE International Workshop on Information Hiding(IH), pp. 51-65. Springer, Berlin,Germany (2010)

57. Shivakumar, B.L., Baboo, S.: Detection of region duplication forgery in digital images using SURF. International Journal of Computer Science Issues 8(4), 199-205 (2011)

58. Shu, L., Fang, Y., Fang, Z., Yang, Y., Fei, F., Xiong, N.: A novel objective quality assessment for super-resolution images. International Journal of Signal Processing 9(5), 297-308 (2016)

59. Silva, E., Carvalho, T., Ferreira, A., Rocha, A.: Going deeper into copy-move forgery detection: Exploring image telltales via multi-scale analysis and voting processes. Journal of Visual Communication and Image Representation 29(C), 16-32 (2015)

60. Vedaldi, A., Fulkerson, B.: Vlfeat: an open and portable library of computer vision algorithms. In: International Conference on Multimedea, pp. 1469-1472. Firenze, Italy (2010)

61. Wang, J., Li, T., Shi, Y.Q., Lian, S., Ye, J.: Forensics feature analysis in quaternion wavelet domain for distinguishing photographic images and computer graphics. Multimedia Tools and Applications 76(22), 1-17 (2016) 
62. Wang, Y., Chen, K., Yu, J., Xiong, N., Leung, H., Zhou, H., Zhu, L.: Dynamic propagation characteristics estimation and tracking based on an em-ekf algorithm in time-variant mimo channel. Information Sciences 408(C), 70-83 (2017)

63. Wang, Z., Fan, B., Wu, F.: Local intensity order pattern for feature description. In: IEEE International Conference on Computer Vision (ICCV), pp. 603-610 (2011)

64. Warif, N.B.A., Wahab, A.W.A., Idris, M.Y.I., Salleh, R., Othman, F.: SIFT-symmetry: A robust detection method for copy-move forgery with reflection attack. Journal of Visual Communication and Image Representation 46, 219-232 (2017)

65. Wu, P., Xiao, F., Sha, C., Huang, H., Wang, R., Xiong, N.: Node scheduling strategies for achieving full-view area coverage in camera sensor networks. Sensors 17(6), 1303 (2017)

66. Xia, Z., Wang, X., Sun, X., Liu, Q., Xiong, N.: Steganalysis of lsb matching using differences between nonadjacent pixels. Multimedia Tools and Applications 75(4), 1947-1962 (2016)

67. Xia, Z., Xiong, N.N., Vasilakos, A.V., Sun, X.: Epcbir: An efficient and privacy-preserving content-based image retrieval scheme in cloud computing. Information Sciences 387, 195$204(2017)$

68. Xiong, N., Jia, X., Yang, L.T., Vasilakos, A.V., Li, Y., Pan, Y.: A distributed efficient flow control scheme for multirate multicast networks. IEEE Transactions on Parallel and Distributed Systems 21(9), 1254-1266 (2010)

69. Xiong, N., Liu, R.W., Liang, M., Wu, D., Liu, Z., Wu, H.: Effective alternating direction optimization methods for sparsity-constrained blind image deblurring. Sensors 17(1), 1-27 (2017)

70. Xiong, N., Vasilakos, A.V., Yang, L.T., Song, L., Pan, Y., Kannan, R., Li, Y.: Comparative analysis of quality of service and memory usage for adaptive failure detectors in healthcare systems. IEEE Journal on Selected Areas in Communications 27(4), 495-509 (2009)

71. Xiong, N., Vasilakos, A.V., Yang, L.T., Wang, C.X., Kannan, R., Chang, C.C., Pan, Y.: A novel self-tuning feedback controller for active queue management supporting tcp flows. Information Sciences 180(11), 2249-2263 (2009)

72. Xu, B., Wang, J., Liu, G., Dai, Y.: Image copy-move forgery detection based on SURF. In: International Conference on Multimedia Information Networking and Security(MINES), pp. 889-892. Nanjing, China (2010)

73. Yang, B., Sun, X., Chen, X., Zhang, J., Li, X.: An efficient forensic method for copy-move forgery detection based on dwt-fwht. Radioengineering 22(4), 1098-1105 (2013)

74. Yang, F., Li, J., Lu, W., Weng, J.: Copy-move forgery detection based on hybrid features. Engineering Applications of Artificial Intelligence 59, 73-83 (2017)

75. Yang, Y., Tong, S., Huang, S., Lin, P.: Dual-tree complex wavelet transform and image block residual-based multi-focus image fusion in visual sensor networks. Sensors 14(12), 22,408-22,430 (2014)

76. Yang, Z., Ma, L., Ma, Q., Cui, J., Nie, Y., Dong, H., An, X.: Multiscale nonlinear thermomechanical coupling analysis of composite structures with quasi-periodic properties. Computers Materials and Continua 53(3), 219-248 (2017)

77. Zhang, C., Wu, D., Liu, R.W., Xiong, N.: Non-local regularized variational model for image deblurring under mixed gaussian-impulse noise. Journal of Internet Technology 16(7), 1301-1319 (2015)

78. Zhang, F., Lu, W., Liu, H., Xue, F.: Natural image deblurring based on 10-regularization and kernel shape optimization. Multimedia Tools and Applications pp. 1-19 (2018)

79. Zhang, H., Liu, R.W., Wu, D., Liu, Y., Xiong, N.N.: Non-convex total generalized variation with spatially adaptive regularization parameters for edge-preserving image restoration. Journal of Internet Technology 17(7), 1391-1403 (2016)

80. Zhang, Q., Lu, W., Wang, R., Li, G.: Digital image splicing detection based on markov features in block dwt domain. Multimedia Tools and Applications pp. 1-22 (2018)

81. Zhang, Q., Lu, W., Weng, J.: Joint image splicing detection in det and contourlet transform domain. Journal of Visual Communication and Image Representation 40, 449-458 (2016)

82. Zhang, Y., Qin, C., Zhang, W., Liu, F., Luo, X.: On the fault-tolerant performance for a class of robust image steganography. Signal Processing 146, 1-1 (2018)

83. Zheng, H., Guo, W., Xiong, N.: A kernel-based compressive sensing approach for mobile data gathering in wireless sensor network systems. IEEE Transactions on Systems Man and Cybernetics Systems PP(99), 1-13 (2017)

84. Zhou, P., Zhou, Y., Wu, D., Jin, H.: Differentially private online learning for cloud-based video recommendation with multimedia big data in social networks. IEEE Transactions on Multimedia 18(6), 1217-1229 (2016) 
85. Zhou, Y., Zhang, D., Xiong, N.: Post-cloud computing paradigms: A survey and comparison. Tsinghua Science and Technology 22(6), 714-732 (2017) 